\title{
Is an elevated hemoglobin concentration a novel risk factor for metabolic syndrome in the Chinese population? a large- scale study
}

\author{
Xiao-Dong Zhou ${ }^{1, *}$, Sheng-Jie Wu ${ }^{1, *}$, Li-Ren Wang ${ }^{2,3}$, Wen-Yue Liu ${ }^{4}$, Ji-Na Zheng ${ }^{2,3}$, \\ Ke-Qing Shi ${ }^{2,5}$, Sven Van Poucke ${ }^{6}$, Dong-Chu Zhang ${ }^{7}$, Wei-Jian Huang ${ }^{1}$, Ming-Hua \\ Zheng $^{2,5}$ \\ ${ }^{1}$ Department of Cardiovascular Medicine, the Heart Center, the First Affiliated Hospital of Wenzhou Medical University, \\ Wenzhou, China \\ ${ }^{2}$ Department of Hepatology, Liver Research Center, the First Affiliated Hospital of Wenzhou Medical University, Wenzhou, \\ China \\ ${ }^{3}$ School of the First Clinical Medical Sciences, Wenzhou Medical University, Wenzhou, China \\ ${ }^{4}$ Department of Endocrinology, the First Affiliated Hospital of Wenzhou Medical University, Wenzhou, China \\ ${ }^{5}$ Institute of Hepatology, Wenzhou Medical University, Wenzhou, China \\ ${ }^{6}$ Department of Anesthesiology, Intensive Care, Emergency Medicine and Pain Therapy, Ziekenhuis Oost-Limburg, Genk, \\ Belgium \\ ${ }^{7}$ Wenzhou Medical Center, Wenzhou People's Hospital, Wenzhou, China \\ *Co-first author \\ Correspondence to: Ming-Hua Zheng, email: zhengmh@wmu.edu.cn \\ Wei-Jian Huang, email: weijianhuang69@126.com
}

Keywords: hemoglobin, metabolic syndrome, risk factor, health examination, longitudinal population

Received: April 10, 2016

Accepted: June 30, 2016

Published: July 13, 2016

\section{ABSTRACT}

Objectives: The potential role of elevated hemoglobin concentration in the development of metabolic syndrome (MS) has not been adequately evaluated. We aim to determine the role of elevated hemoglobin concentrations as a risk factor in MS during health examination of Chinese individuals.

Methods: 57,510 health check-up $(45,451$ by cross-sectional study and 12,059 in a longitudinal population) were conducted. Subjects were divided into quartiles for analyses, using multiple logistic analyses. Furthermore, the logistic analyses were used to explore the association between hemoglobin concentration and individual MS components respectively in the cross-sectional study and the longitudinal study.

Results: In this cross-sectional study, $10.4 \%$ of 45,451 of the health checkup subjects developed MS. Multiple logistic analysis revealed that higher hemoglobin quartiles were positively associated with the development of MS which was in contrast to the lowest hemoglobin quartiles in the fully adjusted model (OR: $2.171,95 \%$ CI $1.944-2.424$ in males, $P<0.001 ; 2.694,95 \%$ CI $2.056-3.531$ in females, $P<0.001$ ). In addition, during a five-year period, $10.8 \%$ of 12,059 health checkup subjects in a longitudinal study were identified. When adjustments were made for compounding factors, the HR for this association between MS and hemoglobin persisted (HR: 2.157, $95 \%$ CI $1.635-2.847$, P $<0.001$ ).

Conclusions: Serum hemoglobin levels were associated with the prevalence of MS in Chinese individuals, suggesting that serum hemoglobin levels might be a novel predictor for the incidence of MS. 


\section{INTRODUCTION}

Metabolic syndrome (MS), characterized by a cluster of individual cardiovascular disease risk factors including hyperlipemia, glucose intolerance, hypertension and central obesity, has recently been proposed to be a key factor leading to a consistent increase in diabetes, cardiovascular disease and even overall mortality [1-3].

Inflammatory biomarkers such as CRP, interleukin-6, tumor necrosis factor- $\alpha$ and white blood cell count have been shown to be related with MS in the context of lowinflammatory states [4-5]. An elevated hemoglobin concentration has also been recognized as a nonconventional risk factor for chronic obstructive pulmonary disease, nonalcoholic fatty liver disease and cardiac disease [6-8]. Moreover, hemoglobin might play an important role in a low-grade inflammatory clinical state [9].

Until now, a few cross-sectional studies have shown the close association between hemoglobin concentrations or red blood cell counts with MS. Hämäläinen et al. revealed that subjects with MS have elevated hemoglobin and erythropoietin (EPO) [10]. Choi et al. and Mardi et al. demonstrated that increased EPO and subclinical inflammation could be part of the MS in Koreans and Europeans [11-12]. Moreover, Kawamoto et al. and Lohsoonthorn et al. also noticed this association in community-dwelling persons in Japan and workers from Bangkok in Thailand respectively [13-14]. However, Kim et al. found no association between red blood cell subtype counts and MS in females [15]. With only 399 participants included, further large-scale studies are desired to clarify the real correlation of hemoglobin concentration with MS. At present, a few longitudinal studies demonstrated the relevance of hemoglobin in the development of MS. Fu et al. provided hematogram models to predict future MS in elderly (above 65 years) in four-year follow-up at the MJ Health Screening Center in Taiwan [16]. Liu et al. also reported similar models with the sensitivity $58.1 \%$ and specificity of $61.4 \%$ in elderly woman [17]. However, the correlation had not been explored for all ages of the population and the association with the individual MS components needs to be elaborated.

Our investigation established a large-scale cross-sectional and a longitudinal study to explore the association between serum hemoglobin concentration and MS (and individual components) and determined whether serum hemoglobin level had a predictive value in the incidence of MS.

\section{RESULTS}

\section{Subject characteristics}

\section{Cross-sectional study}

Baseline clinical and biochemical measurements of 45,451 study participants are summarized in Table 1 . From the entire study population, 4,709 (10.4\%) cases of MS were identified. Compared to the non-MS subjects, the mean age and serum hemoglobin levels were respectively $53.7 \pm 12.9$ years and $147.6 \pm 12.0 \mathrm{~g} / \mathrm{L}$ for the MS subjects and $43.5 \pm$ 14.4 years and $138.7 \pm 14.6 \mathrm{~g} / \mathrm{L}$ in non-MS. As shown in Supplementary Table 1, the incidence of MS is prevalent prominently in males $(14.95 \%$ in males and $3.75 \%$ in females). In order to analyse the influence of gender, the study population was divided into a male and female group. Next, individuals were classified in four groups by hemoglobin level: Q1 ( $\leq 142 \mathrm{~g} / \mathrm{L}$ in males and $\leq 122 \mathrm{~g} / \mathrm{L}$ in female); Q2 (143 - $148 \mathrm{~g} / \mathrm{L}$ in males and $123-128 \mathrm{~g} / \mathrm{L}$ in females); Q3 (149 - $155 \mathrm{~g} / \mathrm{L}$ in male and 129 - $133 \mathrm{~g} / \mathrm{L}$ in females); and Q4 ( $\geq$ $156 \mathrm{~g} / \mathrm{L}$ in males and $\geq 134 \mathrm{~g} / \mathrm{L}$ in females). The incidence of MS increased as distribution of hemoglobin level in quartiles and it was $12.1 \%, 13.4 \%, 14.9 \%, 19.3 \%$ in males, and $1.8 \%$, $2.2 \%, 3.4 \%, 7.0 \%$ in females, respectively. These subjects with higher hemoglobin concentration had higher mean age, male-to-female sex ratio, BMI, SBP, DBP, TG, TC, LDLC, FPG, Hb, RBCC, WBCC, FBG, ALP, GGT, ALT, AST, BUN, sCr and sUA statistically. No significant statistically difference was observed for PLTC and TB between the four quartiles.

\section{Longitudinal study}

From a total of 12059 eligible subjects (mean age 43.8 years, mean hemoglobin $141.6 \mathrm{~g} / \mathrm{L}$ ) with at least three repeated health examination, $10.8 \%$ had MS within the 5-years follow-up periods. Similar details of followup subjects were presented in Supplementary Table S2. Supplementary Table S3 illustrated the relationship between hemoglobin quartiles and the prevalence of MS and its individual components. All individuals were drawn from different hemoglobin levels in the initial year of health examination, and divided into quartiles: $\mathrm{Q} 1, \leq 132$ $\mathrm{g} / \mathrm{L}, \mathrm{n}=3148 ; \mathrm{Q} 2,133-143 \mathrm{~g} / \mathrm{L}, \mathrm{n}=3021 ; \mathrm{Q} 3,144-152$ $\mathrm{g} / \mathrm{L}, \mathrm{n}=3077$ and $\mathrm{Q} 4, \geq 154 \mathrm{~g} / \mathrm{L}, \mathrm{n}=2813$.

\section{Elevated hemoglobin is association with the prevalence of MS}

As observed in Table 2, the multivariate analysis revealed that in the study group of people belonging to the 2-4th hemoglobin level quartiles, the risk of MS was higher with OR 1.112 (95\% CI 1.015 - 1.239), 1.275 (95\% CI $1.161-1.401)$, and 1.740 (95\% CI $1.584-1.911)$ in males, $\mathrm{P}<0.001 ;$ (1.224 (95\% CI $0.919-1.632), 1.907$ (95\% CI 1.454 - 2.501), 4.118 (95\% CI 3.237 - 5.239) in females $\mathrm{P}<0.001$, with the lowest quartile of hemoglobin level considered as a reference in the unadjusted model. After adjustment for age, a higher probability of hemoglobin was directly correlated with MS. In addition, the association also persisted even when adjustment was made to control for age, WBCC, PLTC, ALP, GGT, ALT, AST, BUN, sCr, sUA and TB. (Q4 vs. Q1 2.171, 95\% CI $1.944-2.424$ in males P $<0.001 ; 2.694,95 \%$ CI $2.056-3.531$ in females $\mathrm{P}<0.001$ ) 
Table 1: Baseline characteristics between subjects with and without MS

\begin{tabular}{|c|c|c|c|c|}
\hline Cross-sectional study & Overall & Non-MS & MS & P value \\
\hline $\mathbf{N}$ & $45451(100 \%)$ & $40742(89.6 \%)$ & $4709(10.4 \%)$ & \\
\hline Age (year) & $44.5 \pm 14.6$ & $43.5 \pm 14.4$ & $53.7 \pm 12.9$ & $<0.001$ \\
\hline Male (\%) & $27704(61.0 \%)$ & $23616(58.0 \%)$ & $4088(86.8 \%)$ & $<0.001$ \\
\hline BMI $\left(\mathrm{kg} / \mathrm{m}^{2}\right)$ & $23.1 \pm 3.3$ & $22.7 \pm 3.0$ & $27 \pm 2.4$ & $<0.001$ \\
\hline SBP (mmHg) & $123.2 \pm 17$ & $121 \pm 15.7$ & $143 \pm 14.7$ & $<0.001$ \\
\hline DBP (mmHg) & $76.9 \pm 11.4$ & $75.3 \pm 10.5$ & $90.1 \pm 9.6$ & $<0.001$ \\
\hline TG $(\mathrm{mmol} / \mathrm{L})$ & $1.6 \pm 1.4$ & $1.4 \pm 1.2$ & $2.9 \pm 2.2$ & $<0.001$ \\
\hline $\mathrm{TC}(\mathrm{mmol} / \mathrm{L})$ & $4.7 \pm 0.9$ & $4.6 \pm 0.9$ & $5.2 \pm 1.0$ & $<0.001$ \\
\hline HDL-C (mmol/L) & $1.3 \pm 0.3$ & $1.4 \pm 0.3$ & $1.2 \pm 0.3$ & $<0.001$ \\
\hline LDL-C (mmol/L) & $2.6 \pm 0.7$ & $2.6 \pm 0.7$ & $2.9 \pm 0.7$ & $<0.001$ \\
\hline FBG (mmol/L) & $5.4 \pm 1.1$ & $5.3 \pm 0.8$ & $6.6 \pm 1.9$ & $<0.001$ \\
\hline $\mathrm{Hb}(\mathrm{g} / \mathrm{L})$ & $139.6 \pm 14.6$ & $138.7 \pm 14.6$ & $147.6 \pm 12$ & $<0.001$ \\
\hline $\operatorname{RBCC}\left(10^{3} / \mu \mathrm{L}\right)$ & $4.6 \pm 0.5$ & $4.6 \pm 0.5$ & $4.8 \pm 0.4$ & $<0.001$ \\
\hline WBCC $\left(10^{3} / \mu \mathrm{L}\right)$ & $6.2 \pm 1.6$ & $6.1 \pm 1.5$ & $6.9 \pm 1.6$ & $<0.001$ \\
\hline $\operatorname{PLTC}\left(10^{3} / \mu \mathrm{L}\right)$ & $184.4 \pm 43$ & $184.5 \pm 42.9$ & $183.5 \pm 43.7$ & 0.129 \\
\hline $\operatorname{ALP}(I U / L)$ & $72.4 \pm 23.5$ & $71.6 \pm 23.3$ & $79.6 \pm 23.8$ & $<0.001$ \\
\hline GGT (IU/L) & $32.4 \pm 37.6$ & $29.3 \pm 31.9$ & $59.1 \pm 63.7$ & $<0.001$ \\
\hline ALT (IU/L) & $22 \pm 14.6$ & $21 \pm 13.8$ & $31.3 \pm 18$ & $<0.001$ \\
\hline $\operatorname{AST}(I U / L)$ & $22.8 \pm 8.2$ & $22.3 \pm 7.8$ & $27.1 \pm 10.3$ & $<0.001$ \\
\hline BUN $(\mu \mathrm{mol} / \mathrm{L})$ & $4.6 \pm 1.3$ & $4.5 \pm 1.3$ & $4.9 \pm 1.3$ & $<0.001$ \\
\hline $\mathrm{sCr}(\mu \mathrm{mol} / \mathrm{L})$ & $84.1 \pm 21.5$ & $83.2 \pm 21.1$ & $91.7 \pm 23.8$ & $<0.001$ \\
\hline $\operatorname{sUA}(\mu \mathrm{mol} / \mathrm{L})$ & $312.9 \pm 91.9$ & $306.4 \pm 90.1$ & $369.4 \pm 88.5$ & $<0.001$ \\
\hline TB $(\mu \mathrm{mol} / \mathrm{L})$ & $13.2 \pm 5$ & $13.2 \pm 5$ & $13.3 \pm 4.9$ & 0.164 \\
\hline
\end{tabular}

Abbreviation: MS: metabolic syndrome; BMI: body mass index; SBP: systolic blood pressure; DBP: diastolic blood pressure; TG: triglyceride; TC: total cholesterol; HDL-C: high density lipoprotein-cholesterol; LDL-C: low density lipoprotein-cholesterol; FPG: fasting plasma glucose; Hb: hemoglobin; RBCC: red blood cell counts; WBCC: white blood cell counts; PLTC: platelet count; ALP: alkaline phosphatase; GGT: gamma-glutamyl transferase; ALT: alanine aminotransferase; AST: aspartate aminotransferase; BUN: blood urine nitrogen; sCr: serum creatinine; sUA: serum uric acid; TB: total bilirubin.

\section{Hemoglobin concentration has predictive value for MS}

As illustrated in Table 2, our analyses revealed that initial serum hemoglobin levels were correlated in rude model (HR: 1.0, reference; 2.772, 95\% CI 2.234 - $3.439 ; 4.191,95 \%$ CI $3.410-5.151$ and $4.389,95 \%$ CI 3.566 - 5.402, $\mathrm{P}<0.001$, across increasing quartiles of hemoglobin). Furthermore, after adjustment for age and sex, the association persisted and became more significant. Controlling for all compounding covariates, the highest hemoglobin quartile $(\geq 154 \mathrm{~g} / \mathrm{L})$ are associated with increased odds for MS, compared to the lowest hemoglobin quartile $(\leq 132 \mathrm{~g} / \mathrm{L})$ (HR: 2.157, $95 \%$ CI $1.635-2.847, \mathrm{P}<0.001)$. HRs for each analysis model increased in our trend analysis.

To determine the association with the cumulative prevalence of MS and serum hemoglobin levels, KaplanMeier curves were generated for time to event. As illustrated in Figure 1, baseline serum hemoglobin levels were significantly associated with future incidence of MS, suggesting that increasing levels of serum hemoglobin were progressively associated with an increased incidence of MS. 
Table 2: Quartiles serum hemoglobin concentrations and risk of metabolic syndromes

\begin{tabular}{|c|c|c|c|}
\hline \multirow[t]{2}{*}{ Cross-sectional study } & \multicolumn{3}{|c|}{ Male } \\
\hline & Model A & Model B & Model C \\
\hline Q1 & ref & ref & ref \\
\hline Q2 & $1.122(1.015-1.239)^{\dagger}$ & $1.575(1.419-1.749)^{\dagger}$ & $1.422(1.276-1.584)^{\dagger}$ \\
\hline Q3 & $1.275(1.161-1.401)^{\dagger}$ & $2.031(1.836-2.246)^{\dagger}$ & $1.667(1.500-1.852)^{\dagger}$ \\
\hline Q4 & $1.740(1.584-1.911)^{\dagger}$ & $3.034(2.737-3.363)^{\dagger}$ & $2.171(1.944-2.424)^{\dagger}$ \\
\hline \multirow[t]{2}{*}{ Cross-sectional study } & \multicolumn{3}{|c|}{ Female } \\
\hline & Model A & Model B & Model C \\
\hline Q1 & ref & ref & ref \\
\hline Q2 & $1.224(0.919-1.632)$ & $1.262(0.938-1.697)$ & $1.147(0.842-1.563)$ \\
\hline Q3 & $1.907(1.454-2.501)^{\dagger}$ & $2.027(1.531-2.685)^{\dagger}$ & $1.602(1.192-2.153)^{\dagger}$ \\
\hline Q4 & $4.118(3.237-5.239)^{\dagger}$ & $3.901(3.037-5.012)^{\dagger}$ & $2.694(2.056-3.531)^{\dagger}$ \\
\hline Longitudinal study & Model A & Model B & Model C \\
\hline Q1 & ref & ref & ref \\
\hline Q2 & $2.772(2.234-3.439)^{\dagger}$ & $1.708(1.344-2.171)^{\dagger}$ & $1.615(1.256-2.077)^{\dagger}$ \\
\hline Q3 & $4.191(3.410-5.151)^{\dagger}$ & $2.209(1.721-2.835)^{\dagger}$ & $1.915(1.471-2.493)^{\dagger}$ \\
\hline Q4 & $4.389(3.566-5.402)^{\dagger}$ & $2.694(2.080-3.489)^{\dagger}$ & $2.157(1.635-2.847)^{\dagger}$ \\
\hline
\end{tabular}

Model A is univariate analysis for serum hemoglobin;

Model B is adjusted for age and sex;

Model C is adjusted for age, sex, white blood cell counts, platelet count, alkaline phosphatase, gamma-glutamyl transferase, alanine aminotransferase, aspartate aminotransferase, blood urine nitrogen, serum creatinine, serum uric acid and total bilirubin.

†: $\mathrm{P}<0.05$

\section{Which MS components might exert predominate influence on the association?}

We next repeated our multivariate logistic analyses to further explore the association between hemoglobin and individual MS components in the cross-sectional study (Table 3 \& Figure 2). Three of individual MS components (dyslipdemia, BMI and hypertension) were found to be associated with serum hemoglobin and showed a statistical significance. Using the Q1 as the reference, ORs for dyslipdemia, BMI, hypertension of Q4 were 1.731 (95\% CI 1.589 - 1.885), $\mathrm{P}<0.001 ; 1.390$ (95\% CI 1.277 - 1.513), $\mathrm{P}$ $<0.001 ; 1.347$ (95\% CI $1.231-1.474), \mathrm{P}<0.001$ in males; 1.760 (95\% CI $1.525-2.031), \mathrm{P}<0.001 ; 1.131(95 \% \mathrm{CI}$ 0.976 - 1.312), $\mathrm{P}=0.050 ; 1.754$ (95\% CI 1.496 - 2.056), $\mathrm{P}<$ 0.001 in females. Elevated FPG at an outpatient clinic tended to be higher in patients with MS, compared to non-MS subjects, although the difference was not statistically (Q4 vs. Q1: OR 1.117, 95\% CI 0.997 - 1.253 in males; $1.392,95 \%$ CI 1.139 - 1.701 in females). Moreover, serum hemoglobin levels had no relevance to BMI only in female (Q4 vs. Q1: OR $1.131,95 \%$ CI 0.976 - 1.312). The longitudinal analysis also demonstrated that serum hemoglobin level of all subjects was positively correlated to most of the four individual MS components (Figure 3). However, elevated FPG were not significantly correlated with hemoglobin in all-genders subjects (Q4 vs. Q1: OR 1.188, 95\% CI 0.965 - 1.462).

\section{DISCUSSION}

The study mainly attempted to quantitatively assess the correlation between serum hemoglobin levels and MS. The positive correlation between hemoglobin and MS were not only identified by a cross-sectional study, but were also observed in a longitudinal cohort study. Our findings are in general agreement with previous reports, supporting that three of the individual MS components (dyslipdemia, BMI and hypertension) exerted the predominated effect on the association. $\mathrm{Wu}$ et al. had noticed that RBCC and hemoglobin are associated with MS and might be considered as a potential predictor for the risk of the development of MS on middle-to-upper class urban Han Chinese [18]. However, the research population was derived from the middle-to-upper class and the confounding factors were not adjusted for analysis. 


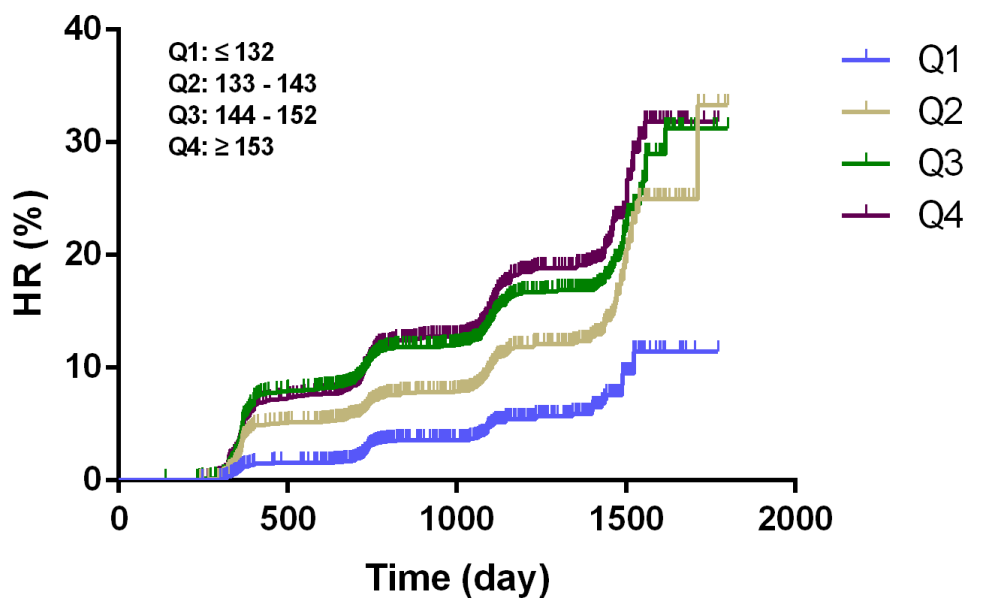

Figure 1: Incidence of metabolic syndrome associated with quartiles of serum hemoglobin in the longitudinal study. All $\mathrm{P}<0.05$

Table 3: Quartiles serum hemoglobin concentrations and risk of four components of metabolic syndromes

\begin{tabular}{|c|c|c|c|c|}
\hline \multirow[t]{2}{*}{ Cross-sectional study } & \multicolumn{4}{|c|}{ Male } \\
\hline & Dyslipdemia* & Hyperglycemia** & BMI*** & Hypertension**** \\
\hline Q1 & ref & ref & ref & ref \\
\hline Q2 & $1.375(1.269-1.489)^{\dagger}$ & $1.064(0.958-1.182)$ & $1.148(1.059-1.244)^{\dagger}$ & $1.043(0.958-1.134)$ \\
\hline Q3 & $1.527(1.412-1.652)^{\dagger}$ & $1.121(1.010-1.244)^{\dagger}$ & $1.237(1.144-1.338)^{\dagger}$ & $1.138(1.047-1.237)^{\dagger}$ \\
\hline Q4 & $1.731(1.589-1.885)^{\dagger}$ & $1.117(0.997-1.253)$ & $1.390(1.277-1.513)^{\dagger}$ & $1.347(1.231-1.474)^{\dagger}$ \\
\hline \multirow[t]{2}{*}{ Cross-sectional study } & \multicolumn{4}{|c|}{ Female } \\
\hline & Dyslipdemia* & Hyperglycemia** & BMI*** & Hypertension**** \\
\hline Q1 & ref & ref & ref & ref \\
\hline Q2 & $1.300(1.124-1.503)^{\dagger}$ & $1.086(0.880-1.339)$ & $1.033(0.890-1.198)$ & $1.299(1.105-1.528)^{\dagger}$ \\
\hline Q3 & $1.420(1.226-1.645)^{\dagger}$ & $1.175(0.952-1.449)$ & $1.075(0.925-1.250)$ & $1.486(1.262-1.750)^{\dagger}$ \\
\hline Q4 & $1.760(1.525-2.031)^{\dagger}$ & $1.392(1.139-1.701)^{\dagger}$ & $1.131(0.976-1.312)$ & $1.754(1.496-2.056)^{\dagger}$ \\
\hline Longitudinal study & Dyslipdemia* & Hyperglycemia** & $\mathbf{B M I} * * *$ & Hypertension $* * * *$ \\
\hline Q1 & ref & ref & ref & ref \\
\hline Q2 & $1.181(1.033-1.350)^{\dagger}$ & $1.140(0.958-1.357)$ & $1.262(1.065-1.496)^{\dagger}$ & $1.233(1.048-1.449)^{\dagger}$ \\
\hline Q3 & $1.396(1.191-1.637)^{\dagger}$ & $1.162(0.958-1.410)$ & $1.493(1.237-1.803)^{\dagger}$ & $1.307(1.087-1.572)^{\dagger}$ \\
\hline Q4 & $1.597(1.344-1.898)^{\dagger}$ & $1.188(0.965-1.462)$ & $1.611(1.320-1.966)^{\dagger}$ & $1.548(1.271-1.885)^{\dagger}$ \\
\hline
\end{tabular}

*: adjusted for age, sex, white blood cell counts, platelet count, alkaline phosphatase, gamma-glutamyl transferase, alanine aminotransferase, aspartate aminotransferase, blood urine nitrogen, serum creatinine, serum uric acid, total bilirubin, systolic blood pressure, diastolic blood pressure, fasting plasma glucose, height and weight.

**: adjusted for age, sex, white blood cell counts, platelet count, alkaline phosphatase, gamma-glutamyl transferase, alanine aminotransferase, aspartate aminotransferase, blood urine nitrogen, serum creatinine, serum uric acid, total bilirubin, triglyceride, high density lipoprotein-cholesterol, systolic blood pressure, diastolic blood pressure, height and weight. ***: adjusted for age, sex, white blood cell counts, platelet count, alkaline phosphatase, gamma-glutamyl transferase, alanine aminotransferase, aspartate aminotransferase, blood urine nitrogen, serum creatinine, serum uric acid, total bilirubin, triglyceride, high density lipoprotein-cholesterol, systolic blood pressure, diastolic blood pressure and fasting plasma glucose. ****: adjusted for age, sex, white blood cell counts, platelet count, alkaline phosphatase, gamma-glutamyl transferase, alanine aminotransferase, aspartate aminotransferase, blood urine nitrogen, serum creatinine, serum uric acid, total bilirubin, triglyceride, high density lipoprotein-cholesterol, fasting plasma glucose, height and weight.

$\dagger: \mathrm{P}<0.05$ 
A

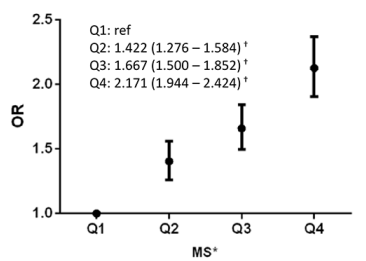

C

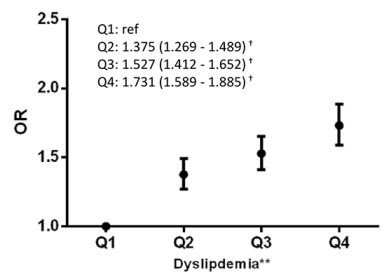

$\mathrm{E}$
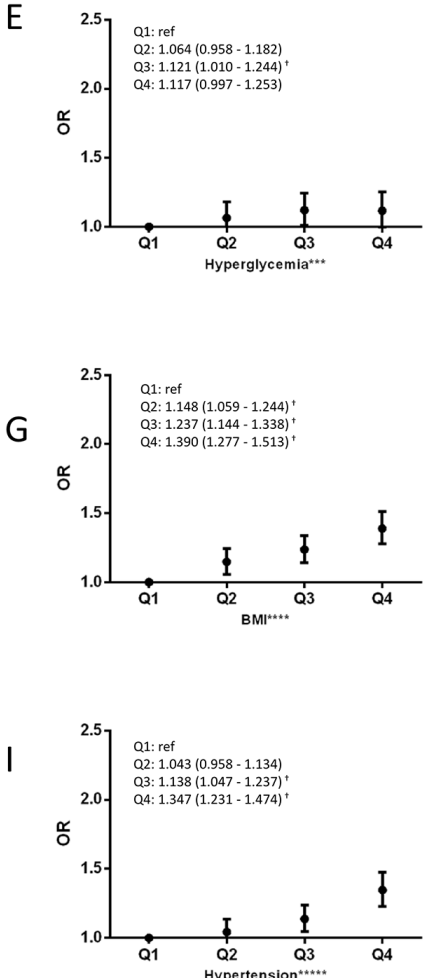

B

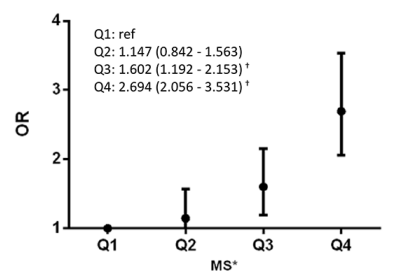

D

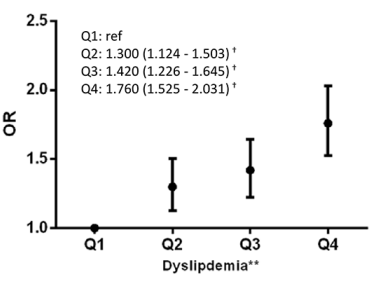

$\mathrm{F}$
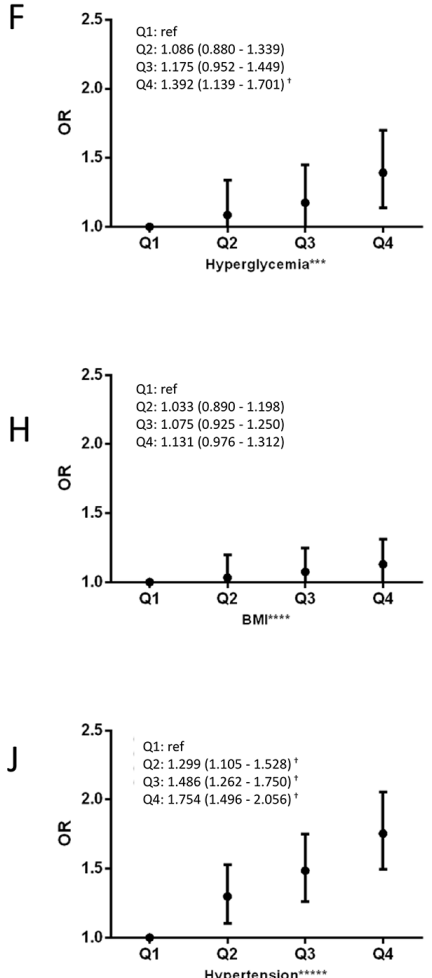

Figure 2: Risks of metabolic syndrome under different status of MS A. and B. dyslipdemia C. and D. hyperglycemia E. and F. BMI $\mathbf{G}$. and $\mathbf{H}$. and hypertension $\mathbf{I}$. and $\mathbf{J}$. associated with quartiles of serum hemoglobin in the cross-sectional study, stratified by genders. *: adjusted for age, sex, white blood cell counts, platelet count, alkaline phosphatase, gamma-glutamyl transferase, alanine aminotransferase, aspartate aminotransferase, blood urine nitrogen, serum creatinine, serum uric acid and total bilirubin **: adjusted for age, sex, white blood cell counts, platelet count, alkaline phosphatase, gamma-glutamyl transferase, alanine aminotransferase, aspartate aminotransferase, blood urine nitrogen, serum creatinine, serum uric acid, total bilirubin, systolic blood pressure, diastolic blood pressure, fasting plasma glucose, height and weight. ***: adjusted for age, sex, white blood cell counts, platelet count, alkaline phosphatase, gamma-glutamyl transferase, alanine aminotransferase, aspartate aminotransferase, blood urine nitrogen, serum creatinine, serum uric acid, total bilirubin, triglyceride, high density lipoprotein-cholesterol, systolic blood pressure, diastolic blood pressure, height and weight. ****: adjusted for age, sex, white blood cell counts, platelet count, alkaline phosphatase, gamma-glutamyl transferase, alanine aminotransferase, aspartate aminotransferase, blood urine nitrogen, serum creatinine, serum uric acid, total bilirubin, triglyceride, high density lipoprotein-cholesterol, systolic blood pressure, diastolic blood pressure and fasting plasma glucose. *****: adjusted for age, sex, white blood cell counts, platelet count, alkaline phosphatase, gamma-glutamyl transferase, alanine aminotransferase, aspartate aminotransferase, blood urine nitrogen, serum creatinine, serum uric acid, total bilirubin, triglyceride, high density lipoprotein-cholesterol, fasting plasma glucose, height and weight. ${ }^{\dagger}: \mathrm{P}<0.05$ 
Although the detailed mechanisms behind the associations of hemoglobin with MS remain not fully resolved, several potential pathogenesic mechanisms have been suggested. First, a review of laboratory research had demonstrated that insulin and insulin growth factors I and II contribute to EPO, both in vitro and in vivo [19-21]. Moreover, the insulin resistance mechanisms, lying at the core of the MS, might be considered as the linkage between elevated hemoglobin level and the progression of MS [19-20,22]. Secondly, the low-grade inflammatory state and endothelial dysfunction may be related with the development of MS [4-5,23-24]. Nitric oxide (NO) -dependent vascular relaxation seems to have a pivotal core role of the insulin-resistant state [25-26]. Various compounds of hemoglobin with NO were previously shown to be positively associated with hemoglobinoxygen affinity of the whole blood, leading to endothelial dysfunction, though the L-arginine-NO pathway $[5,19,25,27]$. Thirdly, higher serum EPO concentrations may suggest underlying adipose tissue hypoxemia in MS $[21,28]$. Several laboratory investigations announced that reduced adipose tissue oxygenation and cellular hypoxia may produce an insulin resistant state, inhibiting insulinstimulated glucose transport activity by preventing the progression of insulin receptor phosphorylation, contributed to MS [29-31]. Several researchers speculated that in essential hypertensive patients, evolution to MS increasing blood viscosity is caused by hemoconcentration
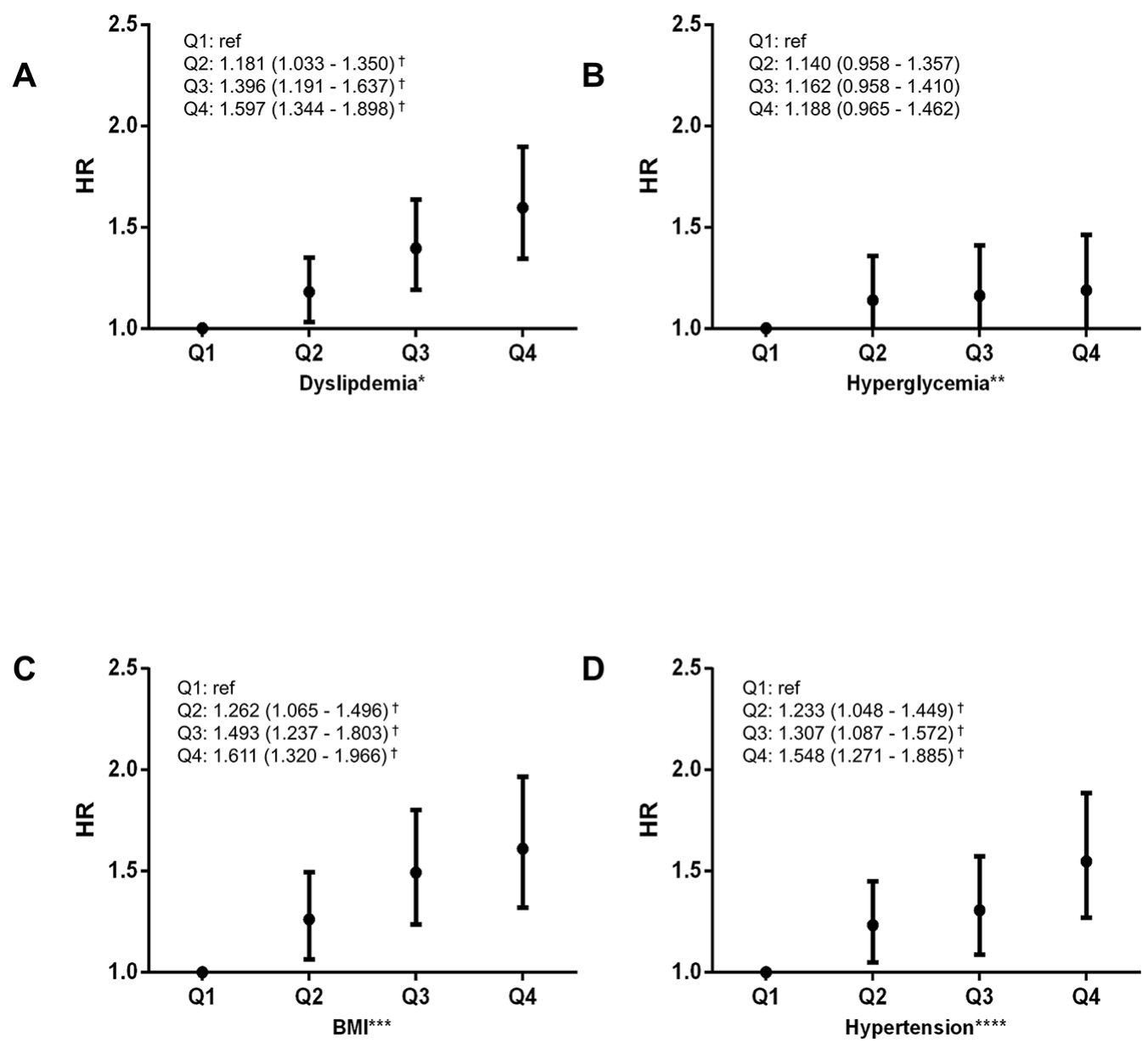

Figure 3: Risks of metabolic syndrome components such as dyslipdemia A. hyperglycemia B. BMI C. and hypertension D. associated with quartiles of serum hemoglobin in the longitudinal study *: adjusted for age, sex, white blood cell counts, platelet count, alkaline phosphatase, gamma-glutamyl transferase, alanine aminotransferase, aspartate aminotransferase, blood urine nitrogen, serum creatinine, serum uric acid, total bilirubin, systolic blood pressure, diastolic blood pressure, fasting plasma glucose, height and weight. **: adjusted for age, sex, white blood cell counts, platelet count, alkaline phosphatase, gamma-glutamyl transferase, alanine aminotransferase, aspartate aminotransferase, blood urine nitrogen, serum creatinine, serum uric acid, total bilirubin, triglyceride, high density lipoproteincholesterol, systolic blood pressure, diastolic blood pressure, height and weight. ***: adjusted for age, sex, white blood cell counts, platelet count, alkaline phosphatase, gamma-glutamyl transferase, alanine aminotransferase, aspartate aminotransferase, blood urine nitrogen, serum creatinine, serum uric acid, total bilirubin, triglyceride, high density lipoprotein-cholesterol, systolic blood pressure, diastolic blood pressure and fasting plasma glucose. $* * * *$ : adjusted for age, sex, white blood cell counts, platelet count, alkaline phosphatase, gammaglutamyl transferase, alanine aminotransferase, aspartate aminotransferase, blood urine nitrogen, serum creatinine, serum uric acid, total bilirubin, triglyceride, high density lipoprotein-cholesterol, fasting plasma glucose, height and weight. ${ }^{\dagger}: \mathrm{P}<0.05$ 
and increased plasma viscosity [24,32-33]. All these above data, support the linkage between hemoglobin and MS. In addition, the serum hemoglobin levels might be a predictive value for the incidence of MS.

A few limitations of the current study deserve consideration. First, potential selection bias might exist, owing to our study participants based on a general health examination population at two Wenzhou health promotion centers. Second, because of insufficient information gathering, several life habits such as smoking, drinking and psychosocial behaviors, which might be a strong determinant of hemoglobin concentrations, were not analyzed in our study. Additionally, he medication history and medical history, as potential confounding factors, were absent in our database. Thus, all of these factors could confound the relationship. Third, owing to the low incidence of MS, especially in females, the relative scarcity of prior study population induced a statistical uncertainty. It is desired to conduct a longitudinal largescale multi-center study in a general population in order to evaluate the association between hemoglobin and the incidence of MS, especially in females.

In summary, serum hemoglobin levels are positively correlated with MS in Chinese population during health examination and might be a potential predictor for the incidence of MS. This might be partially explained because of the individual association of hemoglobin with the three of individual MS components (dyslipdemia, BMI and hypertension).

\section{MATERIALS AND METHODS}

\section{Study population}

Our study population, derived from a cross-sectional and 5-years longitudinal study, was recruited from two separate independent medical centers (Supplementary Figure S1).

In the cross-sectional study, we derived patient data from 45,451 Chinese people (27,704 males and 17,747 females) who attended a routine clinical health examination at the First Affiliated Hospital of Wenzhou Medical University between January 2007 and December 2009. Only subjects with no data of components of MS or hemoglobin were excluded. The current longitudinal study involved the data from a five-year follow-up from the health examination center of the third People's Hospital of Wenzhou from January 2010 to December 2014. Baseline examinations of the longitudinal population were carried out in 2010 and follow-up health examinations were performed every one year since (Supplementary Figure S2). None of the participants met the study exclusion criteria: 1) Less than three repeated health examination within five years; 2) Missing data of examinational date; 3) Missing data of components of MS or hemoglobin.
Finally, a total of 57,510 subjects $(45,451$ in the cross-sectional study and 12,059 in the longitudinal population) were eligible for analysis (Supplementary Figure 1). In addition, all individuals had obtained the detailed health examination. Verbal informed consent was obtained from each subject before their participation in the study. The research protocol of the study was approved by the ethics committee of the First Affiliated Hospital of Wenzhou Medical University and Wenzhou People's Hospital, respectively.

\section{Physical assessment}

Of all subjects, the clinical research coordinators collected physicalassessment data, including age, sex, height and weight, systolic blood pressure (SBP) and diastolic blood pressure (DBP). At every visit, height and weight were measured by a team of trained nurses, by a standard protocol. The body mass index (BMI) was computed by dividing the measured body weight in kilograms $(\mathrm{kg})$ by the square of the measured height in meters (m). Blood pressure was measured with an automatic instrument (Omron, model 705cp, Kyoto, Japan) in seated position after at least a fifteen-minute rest.

\section{Biochemical laboratory test}

Laboratory parameters including hemoglobin concentration, red blood cell counts (RBCC), white blood cell counts (WBCC), triglyceride (TG), total cholesterol (TC), low density lipoprotein-cholesterol (LDL-C), high density lipoprotein-cholesterol (HDL-C), fasting plasma glucose (FPG), serum creatinine $(\mathrm{sCr})$, serum uric acid (sUA), alkaline phosphatase (ALP), aspartate aminotransferase (AST), alanine aminotransferase (ALT) and gamma-glutamyl transferase (GGT) were subsequently analyzed by an automated analyzer (Abbott AxSYM, Park, IL). Blood samples were collected and analyzed from antecubital vein sampling.

\section{Definition}

In this study, MS was diagnosed according to the China Diabetes Federation [34]. Subjects were diagnosed as MS when at least three of the following criteria were satisfied: 1) Central obesity: BMI $\geq 25$ in both genders. 2) Hypertriglyceridemia: $\mathrm{TG} \geq 1.7 \mathrm{mmol} / \mathrm{L}$; HDL-C of $<$ $0.9 \mathrm{mmol} / \mathrm{L}$ in males and $<1.0 \mathrm{mmol} / \mathrm{L}$ in females. 3 ) Hypertension: $\mathrm{SBP} \geq 140 \mathrm{mmHg}$ or $\mathrm{DBP} \geq 90 \mathrm{mmHg}$ or previously diagnosed. 4) Hyperglycemia: FPG $\geq 6.1$ $\mathrm{mmol} / \mathrm{L}$, or hyperglycemia previously diagnosed.

\section{Statistical analysis}

Data analyses were performed with SPSS software (SPSS version 19 for Windows, SPSS software). All clinical data were represented as mean $\pm \mathrm{SD}$ for continuous variables 
and as number (\%) for incidence rates. Statistical significance of continuous variables and categorical variables, were expressed by independent student's T test and Chi-Square tests respectively. In order to assess the correlation of baseline variables with baseline hemoglobin concentrations, the study population was subdivided into subgroups based on the serum hemoglobin concentrations. Multivariable logistic regression analysis was computed to estimate odds ratios (ORs) and 95\% confidence intervals (CI) for exploring the relative risks for each category of hemoglobin level with the incidence of MS. Cox's proportional hazards regression model was performed for the analysis of survival data from longitudinal studies. A two-tailed $\mathrm{P}$ value of $<0.05$ was considered as statistical significance.

\section{Abbreviation}

ALP: alkaline phosphatase; ALT: alanine aminotransferase; AST: aspartate aminotransferase; BMI: body mass index; DBP: diastolic blood pressure; EPO: erythropoietin; FPG: fasting plasma glucose; GGT: gammaglutamyl transferase; HDL-C: high density lipoproteincholesterol; LDL-C: low density lipoprotein-cholesterol; MS: metabolic syndrome; PLTC: platelet count; RBCC: red blood cell counts; SBP: systolic blood pressure; $\mathrm{sCr}$ : serum creatinine; sUA: serum uric acid; TC: total cholesterol;TG: triglyceride; WBCC: white blood cell counts.

\section{ACKNOWLEDGMENTS}

Authors' contributions: Zhou XD, Wu SJ, Huang WJ and Zheng MH designed the study. Zhou XD, Wang LR, Liu WY, Zhang DC collected data. Shi KQ, Zhou XD did the statistical analyses. Liu WY and Zheng JN prepared figures. Zhou XD, Wu SJ, Wang LR, Poucke SV, Huang WJ and Zheng $\mathrm{MH}$ reviewed the results, interpreted data, and wrote the manuscript. All authors have made an intellectual contribution to the manuscript and approved the submission.

\section{CONFLICTS OF INTEREST}

The authors report no declarations of interest.

\section{FUNDING INFORMATION}

This work was supported by grants from National Natural Science Foundation of China (81500665), Zhejiang Engineering Research Center of Intelligent Medicine (2016E10011) and Project of New Century 551 Talent Nurturing in Wenzhou.

\section{REFERENCES}

1. Gami AS, Witt BJ, Howard DE, Erwin PJ, Gami LA, Somers VK, Montori VM. Metabolic syndrome and risk of incident cardiovascular events and death: a systematic review and meta-analysis of longitudinal studies. Journal of the American College of Cardiology. 2007;49:403-414.

2. Sung KC, Rhee EJ, Ryu S, Kim BJ, Kim BS, Lee WY, Oh KW, Kim YB, Chung PW, Kim H, Byrne CD, Lee KB, Park SW. Increased Cardiovascular Mortality in Subjects With Metabolic Syndrome Is Largely Attributable to Diabetes and Hypertension in 159,971 Korean Adults. The Journal of clinical endocrinology and metabolism. 2015;100:2606-2612.

3. Nestel P, Lyu R, Low LP, Sheu WH, Nitiyanant W, Saito I, Tan CE. Metabolic syndrome: recent prevalence in East and Southeast Asian populations. Asia Pacific journal of clinical nutrition. 2007;16:362-367.

4. Esser N, Legrand-Poels S, Piette J, Scheen AJ, Paquot $\mathrm{N}$. Inflammation as a link between obesity, metabolic syndrome and type 2 diabetes. Diabetes research and clinical practice. 2014;105:141-150.

5. Kloting N, Bluher M. Adipocyte dysfunction, inflammation and metabolic syndrome. Reviews in endocrine \& metabolic disorders. 2014;15:277-287.

6. Ding YY, Kader B, Christiansen CL, Berlowitz DR. Hemoglobin Level and Hospital Mortality Among ICU Patients With Cardiac Disease Who Received Transfusions. Journal of the American College of Cardiology. 2015;66:2510-2518.

7. Yu C, Xu C, Xu L, Yu J, Miao M, Li Y. Serum proteomic analysis revealed diagnostic value of hemoglobin for nonalcoholic fatty liver disease. Journal of hepatology. 2012;56:241-247.

8. Ferrari M, Manea L, Anton K, Bruzzone P, Meneghello M, Zamboni F, Purgato L, Cazzoletti L, Ferrari P, Testi R. Anemia and hemoglobin serum levels are associated with exercise capacity and quality of life in chronic obstructive pulmonary disease. BMC pulmonary medicine. 2015;15:58.

9. Alexy T, Pais E, Wenby RB, Mack WJ, Hodis HN, Kono N, Wang J, Baskurt OK, Fisher TC, Meiselman HJ. Abnormal blood rheology and chronic low grade inflammation: possible risk factors for accelerated atherosclerosis and coronary artery disease in Lewis negative subjects. Atherosclerosis. 2015;239:248-251.

10. Hamalainen P, Saltevo J, Kautiainen H, Mantyselka P, Vanhala M. Erythropoietin, ferritin, haptoglobin, hemoglobin and transferrin receptor in metabolic syndrome: a case control study. Cardiovascular diabetology. 2012;11:116.

11. Choi KM, Lee J, Kim YH, Kim KB, Kim DL, Kim SG, Shin DH, Kim NH, Park IB, Choi DS, Baik SH. Relation between insulin resistance and hematological parameters in elderly Koreans-Southwest Seoul (SWS) Study. Diabetes research and clinical practice. 2003;60:205-212.

12. Mardi T, Toker S, Melamed S, Shirom A, Zeltser D, Shapira I, Berliner S, Rogowski O. Increased erythropoiesis and subclinical inflammation as part of the metabolic syndrome. Diabetes research and clinical practice. 2005;69:249-255. 
13. Kawamoto R, Tabara Y, Kohara K, Miki T, Kusunoki T, Abe M, Katoh T. Hematological parameters are associated with metabolic syndrome in Japanese community-dwelling persons. Endocrine. 2013;43:334-341.

14. Lohsoonthorn V, Jiamjarasrungsi W, Williams MA. Association of Hematological Parameters with Clustered Components of Metabolic Syndrome among Professional and Office Workers in Bangkok, Thailand. Diabetes \& metabolic syndrome. 2007;1:143-149.

15. Kim JA, Choi YS, Hong J, Kim SH, Jung HH, Kim SM. Association of metabolic syndrome with white blood cell subtype and red blood cells. Endocrine journal. 2006;53:133-139.

16. Fu YH, Hsu CH, Lin JD, Hsieh CH, Wu CZ, Chao TT, Pei D, Liang YJ, Wang K, Chen YL. Using hematogram model to predict future metabolic syndrome in elderly: a 4-year longitudinal study. The aging male. 2015;18:38-43.

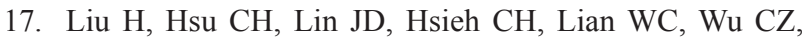
Pei D, Chen YL. Predicting metabolic syndrome by using hematogram models in elderly women. Platelets. 2014;25:97-101.

18. Wu S, Lin H, Zhang C, Zhang Q, Zhang D, Zhang Y, Meng W, Zhu Z, Tang F, Xue F, Liu Y. Association between erythrocyte parameters and metabolic syndrome in urban Han Chinese: a longitudinal cohort study. BMC public health. 2013;13:989.

19. Bersch N, Groopman JE, Golde DW. Natural and biosynthetic insulin stimulates the growth of human erythroid progenitors in vitro. The Journal of clinical endocrinology and metabolism. 1982;55:1209-1211.

20. Miyagawa S, Kobayashi M, Konishi N, Sato T, Ueda K. Insulin and insulin-like growth factor I support the proliferation of erythroid progenitor cells in bone marrow through the sharing of receptors. British journal of haematology. 2000;109:555-562.

21. De Vita F, Maggio M, Lauretani F, Crucitti L, Bandinelli S, Mammarella F, Landi F, Ferrucci L, Ceda GP. INSULINLIKE GROWTH FACTOR-1 AND ANEMIA IN OLDER SUBJECTS: THE INCHIANTI STUDY. Endocrine practice. 2015;21:1211-1218.

22. Widness JA, Susa JB, Garcia JF, Singer DB, Sehgal P, Oh W, Schwartz R, Schwartz HC. Increased erythropoiesis and elevated erythropoietin in infants born to diabetic mothers and in hyperinsulinemic rhesus fetuses. The Journal of clinical investigation. 1981;67:637-642.

23. Wei Y, Liu G, Yang J, Zheng R, Jiang L, Bao P. The association between metabolic syndrome and vascular endothelial dysfunction in adolescents. Experimental and therapeutic medicine. 2013;5:1663-1666.
24. Ucak S, Basat O, Cetinkaya E. Plasma viscosity in patients with metabolic syndrome. Clinical hemorheology and microcirculation. 2013;54:15-22.

25. Kelm M, Rath J. Endothelial dysfunction in human coronary circulation: relevance of the L-arginine-NO pathway. Basic research in cardiology. 2001;96:107-127.

26. Scherrer U, Randin D, Vollenweider P, Vollenweider L, Nicod P. Nitric oxide release accounts for insulin's vascular effects in humans. The Journal of clinical investigation. 1994;94:2511-2515.

27. Pronko TP, Zinchuk VV. Effect of nebivolol on blood oxygen transport indices and endothelial dysfunction in patients with arterial hypertension. Clinical physiology and functional imaging. 2009;29:170-176.

28. Alnaeeli M, Noguchi CT. Erythropoietin and obesityinduced white adipose tissue inflammation: redefining the boundaries of the immunometabolism territory. Adipocyte. 2015;4:153-157.

29. Regazzetti C, Peraldi P, Gremeaux T, Najem-Lendom R, Ben-Sahra I, Cormont M, Bost F, Le Marchand-Brustel Y, Tanti JF, Giorgetti-Peraldi S. Hypoxia decreases insulin signaling pathways in adipocytes. Diabetes. 2009;58:95-103.

30. Pasarica M, Sereda OR, Redman LM, Albarado DC, Hymel DT, Roan LE, Rood JC, Burk DH, Smith SR. Reduced adipose tissue oxygenation in human obesity: evidence for rarefaction, macrophage chemotaxis, and inflammation without an angiogenic response. Diabetes. 2009;58:718-725.

31. Wood IS, de Heredia FP, Wang B, Trayhurn P. Cellular hypoxia and adipose tissue dysfunction in obesity. The Proceedings of the Nutrition Society. 2009;68:370-377.

32. Sugimori H, Tomoda F, Koike T, Kinuno H, Kurosaki $\mathrm{H}$, Masutani $\mathrm{T}$, Inoue H. Blood rheology and platelet function in untreated early-stage essential hypertensives complicated with metabolic syndrome. International journal of hypertension. 2012;2012:109830.

33. Sola E, Vaya A, Simo M, Hernandez-Mijares A, Morillas C, Espana F, Estelles A, Corella D. Fibrinogen, plasma viscosity and blood viscosity in obesity. Relationship with insulin resistance. Clinical hemorheology and microcirculation. 2007;37:309-318.

34. Lu YH, Lu JM, Wang SY, Li CL, Liu LS, Zheng RP, Tian H, Wang XL, Yang LJ, Zhang YQ, Pan CY. [Comparison of the diagnostic criteria of metabolic syndrome by International Diabetes Federation and that by Chinese Medical Association Diabetes Branch]. Zhonghua yi xue za zhi. 2006;86:386-389. 